\title{
Fast Exact Multiplication by the Hessian
}

\author{
Barak A. Pearlmutter \\ Siemens Corporate Research \\ 755 College Road East \\ Princeton, NJ 08540 \\ bap@learning.siemens.com
}

June 9, 1993

To appear in Neural Computation

\begin{abstract}
Just storing the Hessian $\mathbf{H}$ (the matrix of second derivatives $\partial^{2} E / \partial w_{i} \partial w_{j}$ of the error $E$ with respect to each pair of weights) of a large neural network is difficult. Since a common use of a large matrix like $\mathbf{H}$ is to compute its product with various vectors, we derive a technique that directly calculates $\mathbf{H v}$, where $\mathbf{v}$ is an arbitrary vector. To calculate $\mathbf{H v}$, we first define a differential operator $\mathcal{R}_{\mathbf{v}}\{f(\mathbf{w})\}=\left.(\partial / \partial r) f(\mathbf{w}+r \mathbf{v})\right|_{r=0}$, note that $\mathcal{R}_{\mathbf{v}}\left\{\nabla_{\mathbf{W}}\right\}=\mathbf{H v}$ and $\mathcal{R}_{\mathbf{V}}\{\mathbf{w}\}=$ $\mathbf{v}$, and then apply $\mathcal{R}_{\mathbf{V}}\{\cdot\}$ to the equations used to compute $\nabla_{\mathbf{W}}$. The result is an exact and numerically stable procedure for computing $\mathbf{H v}$, which takes about as much computation, and is about as local, as a gradient evaluation. We then apply the technique to a one pass gradient calculation algorithm (backpropagation), a relaxation gradient calculation algorithm (recurrent backpropagation), and two stochastic gradient calculation algorithms (Boltzmann Machines and weight perturbation). Finally, we show that this technique can be used at the heart of many iterative techniques for computing various properties of $\mathbf{H}$, obviating any need to calculate the full Hessian.
\end{abstract}

\section{Introduction}

Efficiently extracting second-order information from large neural networks is an important problem, because properties of the Hessian appear frequently. For instance, in the analysis of the convergence of learning algorithms (Widrow et al., 1979; le Cun et al., 1991; Pearlmutter, 1992); in some techniques for predicting generalization rates in neural networks (MacKay, 1991; Moody, 1992); in techniques for enhancing generalization by weight elimination (le Cun et al., 1990; Hassibi and Stork, 1993); and in full second-order optimization methods (Watrous, 1987).

There exist algorithms for calculating the full Hessian $\mathbf{H}$ (the matrix of second derivative terms $\partial^{2} E / \partial w_{i} \partial w_{j}$ of the error $E$ with respect to the weights w) of a backpropagation network (Bishop, 
1992; Werbos, 1992; Buntine and Weigend, 1991), or reasonable estimates thereof (MacKay, 1991) - but even storing the full Hessian is impractical for large networks. There is also an algorithm for efficiently computing just the diagonal of the Hessian (Becker and le Cun, 1989; le Cun et al., 1990). This is useful when the trace of the Hessian is needed, or when the diagonal approximation is being made-but there is no reason to believe that the diagonal approximation is good in general, and it is reasonable to suppose that, as the system grows, the diagonal elements of the Hessian become less and less dominant. Further, the inverse of the diagonal approximation of the Hessian is known to be a poor approximation to the diagonal of the inverse Hessian.

Here we derive an efficient technique for calculating the product of an arbitrary vector $\mathbf{v}$ with the Hessian $\mathbf{H}$. This allows information to be extracted from the Hessian without ever calculating or storing the Hessian itself. A common use for an estimate of the Hessian is to take its product with various vectors. This takes $O\left(n^{2}\right)$ time when there are $n$ weights. The technique we derive here finds this product in $O(n)$ time and space, ${ }^{1}$ and does not make any approximations.

We first operate in a very general framework, to develop the basic technique. We then apply it to a series of more and more complicated systems, starting with a typical non-iterative gradient calculation algorithm, in particular a backpropagation network, and proceeding to a deterministic relaxation system, and then to some stochastic systems, in particular a Boltzmann Machine and a weight perturbation system.

\section{The Relation Between the Gradient and the Hessian}

The basic technique is to note that the Hessian matrix appears in the expansion of the gradient about a point in weight space,

$$
\nabla_{\mathbf{w}}(\mathbf{w}+\Delta \mathbf{w})=\nabla_{\mathbf{w}}(\mathbf{w})+\mathbf{H} \Delta \mathbf{w}+O\left(\|\Delta \mathbf{w}\|^{2}\right)
$$

where $\mathbf{w}$ is a point in weight space, $\Delta \mathbf{w}$ is a perturbation of $\mathbf{w}, \nabla_{\mathbf{w}}$ is the gradient, the vector of partial derivatives $\partial E / \partial w_{i}$, and $\mathbf{H}$ is the Hessian, the matrix of second derivatives of $E$ with respect to each pair of elements of $\mathbf{w}$. This equation has been used to analyze the convergence properties of some variants of gradient descent (Widrow et al., 1979; le Cun et al., 1991; Pearlmutter, 1992), and to approximate the effect of deleting a weight from the network (le Cun et al., 1990; Hassibi and Stork, 1993). Here we instead use it by choosing $\Delta \mathbf{w}=r \mathbf{v}$, where $\mathbf{v}$ is a vector and $r$ is a small number. We wish to compute $\mathbf{H v}$. Now we note that

$$
\mathbf{H}(r \mathbf{v})=r \mathbf{H v}=\nabla_{\mathbf{w}}(\mathbf{w}+r \mathbf{v})-\nabla_{\mathbf{W}}(\mathbf{w})+O\left(r^{2}\right)
$$

or, dividing by $r$,

$$
\mathbf{H v}=\frac{\nabla_{\mathbf{w}}(\mathbf{w}+r \mathbf{v})-\nabla_{\mathbf{w}}(\mathbf{w})}{r}+O(r) .
$$

This equation provides a simple approximation algorithm for finding $\mathbf{H v}$ for any system whose gradient can be efficiently computed, in time about that required to compute the gradient (assuming

\footnotetext{
${ }^{1} \mathrm{Or} O(p n)$ time when, as is typical for supervised neural networks, the full gradient is the sum of $p$ gradients, each for one single exemplar.
} 
that the gradient at $\mathbf{w}$ has already been computed.) Also, applying the technique requires minimal programming effort. This approximation was used to good effect in (le Cun et al., 1993) and in many numerical analysis optimization routines, which use it to gradually build up an approximation to the inverse Hessian.

Unfortunately, this formula is succeptible to numeric and roundoff problems. The constant $r$ must be small enough that the $O(r)$ term is insignificant. But as $r$ becomes small, large numbers are added to tiny ones in $\mathbf{w}+r \mathbf{v}$, causing a loss of precision of $\mathbf{v}$. A similar loss of precision occurs in the subtraction of the original gradient from the perturbed one, because two nearly identical vectors are being subtracted to obtain the tiny difference between them.

\section{The $\mathcal{R}\{\cdot\}$ Technique}

Fortunately, there is a way to make an algorithm which exactly computes $\mathbf{H v}$, rather than just approximating it, and simultaneously rid ourselves of these numeric difficulties. To do this, we first take the limit of equation (1) as $r \rightarrow 0$. The left hand side stays $\mathbf{H v}$, while the right hand side matches the definition of a derivative, and thus

$$
\mathbf{H v}=\lim _{r \rightarrow 0} \frac{\nabla_{\mathbf{w}}(\mathbf{w}+r \mathbf{v})-\nabla_{\mathbf{w}}(\mathbf{w})}{r}=\left.\frac{\partial}{\partial r} \nabla_{\mathbf{w}}(\mathbf{w}+r \mathbf{v})\right|_{r=0}
$$

As we shall see, there is a simple transformation to convert an algorithm that computes the gradient of the system into one that computes this new quantity. The key to this transformation is to define the operator

$$
\left.\mathcal{R}_{\mathbf{v}}\{f(\mathbf{w})\} \equiv \frac{\partial}{\partial r} f(\mathbf{w}+r \mathbf{v})\right|_{r=0}
$$

so $\mathbf{H v}=\mathcal{R}_{\mathbf{v}}\left\{\nabla_{\mathbf{w}}(\mathbf{w})\right\}$. (To avoid clutter we will usually write $\mathcal{R}\{\cdot\}$ instead of $\mathcal{R}_{\mathbf{v}}\{\cdot\}$.) We can then take all the equations of a procedure that calculates a gradient, e.g. the backpropagation procedure, and we can apply the $\mathcal{R}_{\mathrm{v}}\{\cdot\}$ operator to each equation. Because $\mathcal{R}\{\cdot\}$ is a differential operator, it obeys the usual rules for differential operators, such as:

$$
\begin{aligned}
\mathcal{R}\{c f(\mathbf{w})\} & =c \mathcal{R}\{f(\mathbf{w})\} \\
\mathcal{R}\{f(\mathbf{w})+g(\mathbf{w})\} & =\mathcal{R}\{f(\mathbf{w})\}+\mathcal{R}\{g(\mathbf{w})\} \\
\mathcal{R}\{f(\mathbf{w}) g(\mathbf{w})\} & =\mathcal{R}\{f(\mathbf{w})\} g(\mathbf{w})+f(\mathbf{w}) \mathcal{R}\{g(\mathbf{w})\} \\
\mathcal{R}\{f(g(\mathbf{w}))\} & =f^{\prime}(g(\mathbf{w})) \mathcal{R}\{g(\mathbf{w})\} \\
\mathcal{R}\left\{\frac{d f(\mathbf{w})}{d t}\right\} & =\frac{d \mathcal{R}\{f(\mathbf{w})\}}{d t}
\end{aligned}
$$

Also note that

$$
\mathcal{R}\{\mathbf{w}\}=\mathbf{v} .
$$

These rules are sufficient to derive, from the equations normally used to compute the gradient, a new set of equations about a new set of $\mathcal{R}$-variables. These new equations make use of variables from the original gradient calculation on their right hand sides. This can be thought of as an adjoint 
system to the gradient calculation, just as the gradient calculation of backpropagation can be thought of as an adjoint system to the forward calculation of the error measure. This new adjoint system computes the vector $\mathcal{R}\left\{\nabla_{\mathbf{W}}\right\}$, which is precisely the vector $\mathbf{H v}$ which we desire.

\section{Application of the $\mathcal{R}\{\cdot\}$ Technique to Various Networks}

Let us utilize this new technique for transforming the equations that compute the gradient into equations that compute $\mathbf{H v}$, the product of a vector $\mathbf{v}$ with the Hessian $\mathbf{H}$. We will, rather mechanically, derive appropriate algorithms for some standard sorts of neural networks that typify three broad classes of gradient calculation algorithms. These examples are intended to be illustrative, as the technique applies equally well to most other gradient calculation procedures.

Usually the error $E$ is the sum of the errors for many patterns, $E=\sum_{p} E_{p}$. Therefore $\nabla_{\mathbf{w}}$ and $\mathbf{H}$ are sums over all the patterns, $\mathbf{H}=\sum_{p} \mathbf{H}_{p}$, and $\mathbf{H v}=\sum_{p} \mathbf{H}_{p} \mathbf{v}$. As is usual, for clarity this outer sum over patterns is not shown except where necessary, and the gradient and $\mathbf{H v}$ procedures are shown for only a single exemplar.

\subsection{Simple Backpropagation Networks}

Let us apply the above procedure to a simple backpropagation network, to derive the $\mathcal{R}\{$ backprop $\}$ algorithm, a set of equations that can be used to efficiently calculate $\mathbf{H v}$ for a backpropagation network. The $\mathcal{R}\{$ backprop\} algorithm was independently discovered by (Werbos, 1988, eq. 14), who derived it as a backpropagation process to calculate $\mathbf{H v}=\nabla_{\mathbf{w}}\left(\mathbf{v} \cdot \nabla_{\mathrm{w}} E\right)$, where $\nabla_{\mathbf{w}} E$ is also calculated by backpropagation. Interestingly, that derivation is dual to the one given here, in that the direction of the equations is reversed, the backwards pass of the $\nabla_{\mathrm{w}} E$ algorithm becoming a forward pass in the $\mathbf{H v}$ algorithm, while here the direction of the equations is unchanged. The same algorithm was also discovered, with yet another derivation, by (Møller, 1993a).

For convenience, we will now change our notation for indexing the weights $\mathbf{w}$. Let $\mathbf{w}$ be the weights, now doubly indexed by their source and destination units' indices, as in $w_{i j}$, the weight from unit $i$ to unit $j$. Because $\mathbf{v}$ is of the same dimension as $\mathbf{w}$, its elements will be similarly indexed. All sums over indices are limited to weights that exist in the network topology. As is usual, quantities which occur on the left sides of the equations are treated computationally as variables, and calculated in topological order, which is assumed to exist because the weights, regarded as a connection matrix, is zero-diagonal and can be put into triangular form (Werbos, 1974).

The forward computation of the network is ${ }^{2}$

$$
x_{i}=\sum_{j} w_{j i} y_{j}
$$

\footnotetext{
${ }^{2}$ This compact form of the backpropagation equations, due to Fernando Pineda, unifies the special cases of input units, hidden units, and output units. In the case of a unit $i$ with no incoming weights, i.e. an input unit, it simplifies to $y_{i}=\sigma_{i}(0)+I_{i}$, allowing the value to be set entirely externally. For a hidden unit or output $i$, the term $I_{i}=0$. In the corresponding equations for the backward pass (7) only the output units have nonzero direct error terms $e_{i}$, and since such output units have no outgoing weights, the situation for an output unit $i$ simplifies to $\partial E / \partial y_{i}=e_{i}\left(y_{i}\right)$.
} 


$$
y_{i}=\sigma_{i}\left(x_{i}\right)+I_{i}
$$

where $\sigma_{i}(\cdot)$ is the nonlinearity of the $i^{t h}$ unit, $x_{i}$ is the total input to the $i^{t h}$ unit, $y_{i}$ is the output of the $i^{\text {th }}$ unit, and $I_{i}$ is the external input (from outside the network) to the $i^{t h}$ unit.

Let the error measure be $E=E(y)$, and its simple direct derivative with respect to $y_{i}$ be $e_{i}=d E / d y_{i}$. We assume that $e_{i}$ depends only on $y_{i}$, and not on any $y_{j}$ for $j \neq i$. This is true of most common error measures, such as squared error or cross entropy (Hinton, 1987). ${ }^{3}$ We can thus write $e_{i}\left(y_{i}\right)$ as a simple function. The backward pass is then

$$
\begin{aligned}
\frac{\partial E}{\partial y_{i}} & =e_{i}\left(y_{i}\right)+\sum_{j} w_{i j} \frac{\partial E}{\partial x_{j}} \\
\frac{\partial E}{\partial x_{i}} & =\sigma_{i}^{\prime}\left(x_{i}\right) \frac{\partial E}{\partial y_{i}} \\
\frac{\partial E}{\partial w_{i j}} & =y_{i} \frac{\partial E}{\partial x_{j}}
\end{aligned}
$$

Applying $\mathcal{R}\{\cdot\}$ to the above equations gives

$$
\begin{aligned}
& \mathcal{R}\left\{x_{i}\right\}=\sum_{j}\left(w_{j i} \mathcal{R}\left\{y_{j}\right\}+v_{j i} y_{j}\right) \\
& \mathcal{R}\left\{y_{i}\right\}=\mathcal{R}\left\{x_{i}\right\} \sigma_{i}^{\prime}\left(x_{i}\right)
\end{aligned}
$$

for the forward pass, and, for the backward pass,

$$
\begin{aligned}
\mathcal{R}\left\{\frac{\partial E}{\partial y_{i}}\right\} & =e_{i}^{\prime}\left(y_{i}\right) \mathcal{R}\left\{y_{i}\right\}+\sum_{j}\left(w_{i j} \mathcal{R}\left\{\frac{\partial E}{\partial x_{j}}\right\}+v_{i j} \frac{\partial E}{\partial x_{j}}\right) \\
\mathcal{R}\left\{\frac{\partial E}{\partial x_{i}}\right\} & =\sigma_{i}^{\prime}\left(x_{i}\right) \mathcal{R}\left\{\frac{\partial E}{\partial y_{i}}\right\}+\mathcal{R}\left\{x_{i}\right\} \sigma_{i}^{\prime \prime}\left(x_{i}\right) \frac{\partial E}{\partial y_{i}} \\
\mathcal{R}\left\{\frac{\partial E}{\partial w_{i j}}\right\} & =y_{i} \mathcal{R}\left\{\frac{\partial E}{\partial x_{j}}\right\}+\mathcal{R}\left\{y_{i}\right\} \frac{\partial E}{\partial x_{j}}
\end{aligned}
$$

The vector whose elements are $\mathcal{R}\left\{\partial E / \partial w_{i j}\right\}$ is just $\mathcal{R}\left\{\nabla_{\mathbf{w}}\right\}=\mathbf{H v}$, the quantity we wish to compute.

For sum squared error $e_{i}\left(y_{i}\right)=y_{i}-d_{i}$ where $d_{i}$ is the desired output for unit $i$, so $e_{i}^{\prime}\left(y_{i}\right)=1$. This simplifies (9) for simple output units to $\mathcal{R}\left\{\partial E / \partial y_{i}\right\}=\mathcal{R}\left\{y_{i}\right\}$. Note that, in the above equations, the topology of the neural network sometimes results in some $\mathcal{R}$-variables being guaranteed to be zero when $\mathbf{v}$ is sparse - in particular when $\mathbf{v}=\left(\begin{array}{llllll}0 & \cdots & 1 & 0 & \cdots & 0\end{array}\right)$, which can be used to compute a single desired column of the Hessian. In this situation, some of the computation is also shared between various columns.

${ }^{3}$ If this assumption is violated then in equation (9) the $e_{i}^{\prime}\left(y_{i}\right) \mathcal{R}\left\{y_{i}\right\}$ term generalizes to $\sum_{j}\left(\partial e_{i} / \partial y_{j}\right) \mathcal{R}\left\{y_{j}\right\}$. 


\subsection{Recurrent Backpropagation Networks}

The recurrent backpropagation algorithm (Almeida, 1987; Pineda, 1987) consists of a set of forward equations which relax to a solution for the gradient,

$$
\begin{aligned}
x_{i} & =\sum_{j} w_{j i} y_{j} \\
\frac{d y_{i}}{d t} & \propto-y_{i}+\sigma_{i}\left(x_{i}\right)+I_{i} \\
\frac{d z_{i}}{d t} & \propto-z_{i}+\sigma_{i}^{\prime}\left(x_{i}\right) \sum_{j}\left(w_{i j} z_{j}\right)+e_{i}\left(y_{i}\right) \\
\frac{\partial E}{\partial w_{i j}} & =\left.y_{i} z_{j}\right|_{t=\infty}
\end{aligned}
$$

Adjoint equations for the calculation of $\mathbf{H v}$ are obtained by applying the $\mathcal{R}\{\cdot\}$ operator, yielding

$$
\begin{aligned}
\mathcal{R}\left\{x_{i}\right\} & =\sum_{j}\left(w_{j i} \mathcal{R}\left\{y_{i}\right\}+v_{j i} y_{j}\right) \\
\frac{d \mathcal{R}\left\{y_{i}\right\}}{d t} & \propto-\mathcal{R}\left\{y_{i}\right\}+\sigma_{i}^{\prime}\left(x_{i}\right) \mathcal{R}\left\{x_{i}\right\} \\
\frac{d \mathcal{R}\left\{z_{i}\right\}}{d t} & \propto-\mathcal{R}\left\{z_{i}\right\}+\sigma_{i}^{\prime}\left(x_{i}\right) \sum_{j}\left(v_{i j} z_{j}+w_{i j} \mathcal{R}\left\{z_{j}\right\}\right)+\sigma_{i}^{\prime \prime}\left(x_{i}\right) \mathcal{R}\left\{x_{i}\right\} \sum_{j}\left(w_{i j} z_{j}\right)+e_{i}^{\prime}\left(y_{i}\right) \mathcal{R}\left\{y_{i}\right\} \\
\mathcal{R}\left\{\frac{\partial E}{\partial w_{i j}}\right\} & =y_{i} \mathcal{R}\left\{z_{j}\right\}+\left.\mathcal{R}\left\{y_{i}\right\} z_{j}\right|_{t=\infty}
\end{aligned}
$$

These equations specify a relaxation process for computing $\mathbf{H v}$. Just as the relaxation equations for computing $\nabla_{\mathrm{W}}$ are linear even though those for computing $y$ and $E$ are not, these new relaxation equations are linear.

\subsection{Stochastic Boltzmann Machines}

One might ask whether this technique can be used to derive a Hessian multiplication algorithm for a classic Boltzmann Machine (Ackley et al., 1985), which is discrete and stochastic, unlike its continuous and deterministic cousin to which application of $\mathcal{R}\{\cdot\}$ is simple. A classic Boltzmann Machine operates stochastically, with its binary unit states $s_{i}$ taking on random values according to the probability

$$
\begin{aligned}
P\left(s_{i}=1\right) & =p_{i}=\sigma\left(x_{i} / T\right) \\
x_{i} & =\sum_{j} w_{j i} s_{j}
\end{aligned}
$$

At equilibrium, the probability of a state $\alpha$ of all the units (not just the visible units) is related to its energy

$$
E_{\alpha}=\sum_{i<j} s_{i}^{\alpha} s_{j}^{\alpha} w_{i j}
$$


by $P(\alpha)=Z^{-1} \exp -E_{\alpha} / T$, where the partition function is $Z=\sum_{\alpha} \exp -E_{\alpha} / T$. The system's equilibrium statistics are sampled because, at equilibrium,

$$
\frac{\partial G}{\partial w_{i j}}=\left(p_{i j}^{+}-p_{i j}^{-}\right) / T
$$

where $p_{i j}=\left\langle s_{i} s_{j}\right\rangle, G$ is the asymmetric divergence, an information theoretic measure of the difference between the environmental distribution over the output units and that of the network, as used in (Ackley et al., 1985), $T$ is the temperature, and the + and - superscripts indicate the environmental distribution, + for waking and - for hallucinating.

Applying the $\mathcal{R}\{\cdot\}$ operator, we obtain

$$
\mathcal{R}\left\{\frac{\partial G}{\partial w_{i j}}\right\}=\left(\mathcal{R}\left\{p_{i j}^{+}\right\}-\mathcal{R}\left\{p_{i j}^{-}\right\}\right) / T .
$$

We shall soon find it useful if we define

$$
\begin{aligned}
D_{\alpha} & =\mathcal{R}\left\{E_{\alpha}\right\}=\sum_{i<j} s_{i}^{\alpha} s_{j}^{\alpha} v_{i j} \\
q_{i j} & =\left\langle s_{i} s_{j} D\right\rangle
\end{aligned}
$$

(with the letter $D$ chosen because it has the same relation to $\mathbf{v}$ that $E$ has to $\mathbf{w}$ ) and to note that

$$
\langle D\rangle=\sum_{i<j} p_{i j} v_{i j}
$$

With some calculus, we find $\mathcal{R}\left\{\exp -E_{\alpha} / T\right\}=-P(\alpha) Z D_{\alpha} / T$, and thus $\mathcal{R}\{Z\}=-Z\langle D\rangle / T$. Using these and the relation between the probability of a state and its energy, we have

$$
\mathcal{R}\{P(\alpha)\}=P(\alpha)\left(\langle D\rangle-D_{\alpha}\right) / T
$$

where the expression $P(\alpha)$ can not be treated as a constant because it is defined over all the units, not just the visible ones, and therefore depends on the weights. This can be used to calculate

$$
\begin{aligned}
\mathcal{R}\left\{p_{i j}\right\} & =\sum_{\alpha} \mathcal{R}\{P(\alpha)\} s_{i}^{\alpha} s_{j}^{\alpha} \\
& =\sum_{\alpha} P(\alpha)\left(\langle D\rangle-D_{\alpha}\right) s_{i}^{\alpha} s_{j}^{\alpha} / T \\
& =\left(\left\langle s_{i} s_{j}\right\rangle\langle D\rangle-\left\langle s_{i} s_{j} D\right\rangle\right) / T \\
& =\left(p_{i j}\langle D\rangle-q_{i j}\right) / T .
\end{aligned}
$$

This beautiful formula ${ }^{4}$ gives an efficient way to compute Hv for a Boltzmann Machine, or at least as efficient a way as is used to compute the gradient, simply by using sampling to estimate $q_{i j}$. This requires the additional calculation and broadcast of the single global quantity $D$, but is otherwise local.

The collection of statistics for the gradient is sometimes accelerated by using the equation

$$
p_{i j}=\left\langle s_{i}\right\rangle\left\langle s_{j} \mid s_{i}=1\right\rangle=\left\langle p_{i}\right\rangle\left\langle p_{j} \mid s_{i}=1\right\rangle .
$$

\footnotetext{
${ }^{4}$ Equation (20) is similar in form to that of the gradient of the entropy (Geoff Hinton, personal communication.)
} 
The analogous identity for accelerating the computation of $q_{i j}$ is

$$
q_{i j}=\left\langle p_{i}\right\rangle\left\langle s_{j} D \mid s_{i}=1\right\rangle
$$

or

$$
q_{i j}=\left\langle p_{i}\right\rangle\left\langle p_{j}\left(D+\left(1-s_{j}\right) \Delta D_{j}\right) \mid s_{i}=1\right\rangle
$$

where $\Delta D_{i}=\sum_{j} s_{j} v_{j i}$ is defined by analogy with $\Delta E_{i}=\left.E\right|_{s_{i}=1}-\left.E\right|_{s_{i}=0}=\sum_{j} s_{j} w_{j i}$.

The derivation here was for the simplest sort of Boltzmann Machine, with binary units and only pairwise connections between the units. However, the technique is immediately applicable to higher order Boltzmann Machines (Hinton, 1987), as well as to Boltzmann Machines with non-binary units (Movellan and McClelland, 1991).

\subsection{Weight Perturbation}

In weight perturbation (Jabri and Flower, 1991; Alspector et al., 1993; Flower and Jabri, 1993; Kirk et al., 1993; Cauwenberghs, 1993) the gradient $\nabla_{\mathbf{W}}$ is approximated using only the globally broadcast result of the computation of $E(\mathbf{w})$. This is done by adding a random zero-mean perturbation vector $\Delta \mathbf{w}$ to $\mathbf{w}$ repeatedly and approxmating the resulting change in error by

$$
E(\mathbf{w}+\Delta \mathbf{w})=E(\mathbf{w})+\Delta E=E(\mathbf{w})+\nabla_{\mathbf{w}} \cdot \Delta \mathbf{w} .
$$

From the viewpoint of each individual weight $w_{i}$

$$
\Delta E=\Delta w_{i} \frac{\partial E}{\partial w_{i}}+\text { noise }
$$

Because of the central limit theorem it is reasonable to make a least-squares estimate of $\partial E / \partial w_{i}$, which is $\partial E / \partial w_{i}=\left\langle\Delta w_{i} \Delta E\right\rangle /\left\langle\Delta w_{i}^{2}\right\rangle$. The numerator is estimated from corresponding samples of $\Delta w_{i}$ and $\Delta E$, and the denominator from prior knowledge of the distribution of $\Delta w_{i}$. This requires only the global broadcast of $\Delta E$, while each $\Delta w_{i}$ can be generated and used locally.

It is hard to see a way to mechanically apply $\mathcal{R}\{\cdot\}$ to this procedure, but we can nonetheless derive a suitable procedure for estimating $\mathbf{H v}$. We note that a better approximation for the change in error would be

$$
E(\mathbf{w}+\Delta \mathbf{w})=E(\mathbf{w})+\nabla_{\mathbf{w}} \cdot \Delta \mathbf{w}+\frac{1}{2} \Delta \mathbf{w}^{T} \hat{\mathbf{H}} \Delta \mathbf{w}
$$

where $\widehat{\mathbf{H}}$ is an estimate of the Hessian $\mathbf{H}$. We wish to include in $\widehat{\mathbf{H}}$ only those properties of $\mathbf{H}$ which are relevent. Let us define $\mathbf{z}=\mathbf{H v}$. If $\widehat{\mathbf{H}}$ is to be small in the least squares sense, but also $\widehat{\mathbf{H}} \mathbf{v}=\mathbf{z}$, then the best choice would then be $\widehat{\mathbf{H}}=\mathbf{z v}^{T} /\|\mathbf{v}\|^{2}$, except that then $\widehat{\mathbf{H}}$ would not be symmetric, and therefore the error surface would not be well defined. Adding the symmetry requirement, which is amounts to the added constraint $\mathbf{v}^{T} \hat{\mathbf{H}}=\mathbf{z}^{T}$, the least squares $\widehat{\mathbf{H}}$ becomes

$$
\widehat{\mathbf{H}}=\frac{1}{\|\mathbf{v}\|^{2}}\left(\mathbf{z} \mathbf{v}^{T}+\mathbf{v} \mathbf{z}^{T}-\frac{\mathbf{v} \cdot \mathbf{z}}{\|\mathbf{v}\|^{2}} \mathbf{v} \mathbf{v}^{T}\right) \text {. }
$$

Substituting this in and rearranging the terms, we find that, from the perspective of each weight,

$$
\Delta E=\Delta w_{i} \frac{\partial E}{\partial w_{i}}+\frac{\Delta \mathbf{w} \cdot \mathbf{v}}{\|\mathbf{v}\|^{2}}\left(\Delta w_{i}-\frac{\Delta \mathbf{w} \cdot \mathbf{v}}{2\|\mathbf{v}\|^{2}} v_{i}\right) z_{i}+\text { noise } .
$$


This allows both $\partial E / \partial w_{i}$ and $z_{i}$ to be estimated in the same least-squares fashion as above, using only locally available values, $v_{i}$ and $\Delta w_{i}$, and the globally broadcast $\Delta E$, plus a new quantity which must be computed and globally broadcast, $\Delta \mathbf{w} \cdot \mathbf{v}$. The same technique applies equally well to other perturbative procedures, such as unit perturbation (Flower and Jabri, 1993), and a similar derivation can be used to find the diagonal elements of $\mathbf{H}$, without the need for any additional globally broadcast values.

\section{Practical Applications}

The $\mathcal{R}\{\cdot\}$ technique makes it possible to calculate $\mathbf{H v}$ efficiently. This can be used in the center of many different iterative algorithms, in order to extract particular properties of $\mathbf{H}$. In essence, it allows $\mathbf{H}$ to be treated as a generalized sparse matrix.

\subsection{Finding Eigenvalues and Eigenvectors}

Standard variants of the power method allow one to

- Find the largest few eigenvalues of $\mathbf{H}$, and their eigenvectors.

- Find the smallest few eigenvalues of $\mathbf{H}$, and their eigenvectors.

- Sample H's eigenvalue spectrum, along with the corresponding eigenvectors.

The clever algorithm of (Skilling, 1989) estimates the eigenvalue spectrum of a generalized sparse matrix. It starts by choosing a random vector $\mathbf{v}_{0}$, calculating $\mathbf{v}_{i}=\mathbf{H}^{i} \mathbf{v}_{0}$ for $i=1, \ldots, m$, using the dot products $\mathbf{v}_{i} \cdot \mathbf{v}_{j}$ as estimates of the moments of the eigenvalue spectrum, and using these moments to recover the shape of the eigenvalue spectrum. This algorithm is made applicable to the Hessian by the $\mathcal{R}\{\cdot\}$ technique, in both deterministic and, with minor modifications, stochastic gradient settings.

\subsection{Multiplication by the Inverse Hessian}

It is frequently necessary to find $\mathbf{x}=\mathbf{H}^{-1} \mathbf{b}$, which is the key calculation of all Newton's-method second-order numerical optimization techniques, and is also used in the Optimal Brain Surgeon technique, and in some techniques for predicting the generalization rate. The $\mathcal{R}\{\cdot\}$ technique does not directly solve this problem, but instead one can solve $\mathbf{H} \mathbf{x}=\mathbf{b}$ for $\mathbf{x}$ by minimizing $\|\mathbf{H x}-\mathbf{b}\|^{2}$ using the conjugate-gradient method, thus exactly computing $\mathbf{x}=\mathbf{H}^{-1} \mathbf{b}$ in $n$ iterations without calculating or storing $\mathbf{H}^{-1}$. This squares the condition number, but if $\mathbf{H}$ is known to be positive definite, one can instead minimize $\mathbf{x}^{T} \mathbf{H x} / 2+\mathbf{x} \cdot \mathbf{b}$, which does not square the condition number (Press et al., 1988, page 78). This application of fast exact multiplication by the Hessian, in particular $\mathcal{R}\{$ backprop\}, was independently noted in (Werbos, 1988). 


\subsection{Step Size and Line Search}

Many optimization techniques repeatedly choose a direction $\mathbf{v}$, and then proceed along that direction some distance $\mu$, which takes the system to the constrained minimum of $E(\mathbf{w}+\mu \mathbf{v})$. Finding the value for $\mu$ which minimizes $E$ is called a line search, because it searches only along the line $\mathbf{w}+\mu \mathbf{v}$. There are many techniques for performing a line search. Some are approximate while others attempt to find an exact constrained minimum, and some use only the value of the error, while others also make use of the gradient.

In particular, the line search used within the Scaled Conjugate Gradient (SCG) optimization procedure, in both its deterministic (Møller, 1993b) and stochastic (Møller, 1993c) incarnations, makes use of both first- and second-order information at $\mathbf{w}$ to determine how far to move. The first order information used is simply $\nabla_{\mathbf{w}}(\mathbf{w})$, while the second-order information is precisely Hv, calculated with the one-sided finite difference approximation of equation (1). It can thus benefit immediately from the exact calculation of $\mathbf{H v}$. In fact, the $\mathcal{R}\{$ backprop $\}$ procedure was independently discovered for that application (Møller, 1993a).

The SCG line search proceeds as follows. Assuming that the error $E$ is well approximated by a quadratic, then the product $\mathbf{H v}$ and the gradient $\nabla_{\mathbf{W}}(\mathbf{w})$ predicts of the gradient at any point along the line $\mathbf{w}+\mu \mathrm{v}$ by

$$
\nabla_{\mathbf{w}}(\mathbf{w}+\mu \mathbf{v})=\nabla_{\mathbf{w}}(\mathbf{w})+\mu \mathbf{H v}+O\left(\mu^{2}\right) .
$$

Disregarding the $O\left(\mu^{2}\right)$ term, if we wish to choose $\mu$ to minimize the error, we take the dot product of $\nabla_{\mathbf{w}}(\mathbf{w}+\mu \mathbf{v})$ with $\mathbf{v}$ and set it equal to zero, as the gradient at the constrained minimum must be orthogonal to the space under consideration. This gives $\mathbf{v} \cdot \nabla_{\mathbf{w}}(\mathbf{w})+\mu \mathbf{v}^{T} \mathbf{H v}=0$ or

$$
\mu=-\frac{\mathbf{v} \cdot \nabla_{\mathbf{w}}(\mathbf{w})}{\mathbf{v}^{T} \mathbf{H} \mathbf{v}}
$$

Equation (28) then gives a prediction of the gradient at $\mathbf{w}+\mu \mathbf{v}$. To access the accuracy of the quadratic approximation we might wish to compare this with a gradient measurement taken at that point, or we might even preemptively take a step in that direction.

\subsection{Eigenvalue Based Learning Rate Optimization for Stochastic Gradient Descent}

The technique descibed in the previous section is, at least as stated, suitable only for deterministic gradient descent. In many systems, particularly large ones, deterministic gradient descent is impractical; only noisy estimates of the gradient are available. In joint work with colleagues at AT\&T Bell Labs (le Cun et al., 1993), the approximation technique of equation (1) enabled $\mathbf{H}$ to be treated as a generalized sparse matrix, and properties of $\mathbf{H}$ were extracted in order to accelerate the convergence of stochastic gradient descent.

Information accumulated online, in particular eigenvalues and eigenvectors of the principle eigenspace, was used to linearly transform the weight space in such a way that the ill-conditioned off-axis long narrow valleys in weight space, which slow down gradient descent, become wellconditioned circular bowls. This work did not use an exact value for $\mathbf{H v}$, but rather a stochastic unbiased estimate of the Hessian based on just a single exemplar at a time. Computations of the 
form $\mathbf{x}(t)=\mathbf{H}(t) \mathbf{v}$ were replaced with relaxations of the form $\mathbf{x}(t)=(1-\alpha) \mathbf{x}(t-1)+\alpha \widehat{\mathbf{H}}(t) \mathbf{v}$, where $0<\alpha \ll 1$ determines the trade-off between steady-state noise and speed of convergence.

\section{Summary and Conclusion}

Second-order information about the error is of great practical and theoretical importance. It allows sophisticated optimization techniques to be applied, appears in many theories of generalization, and is used in sophisticated weight pruning procedures. Unfortunately, the Hessian matrix $\mathbf{H}$, whose elements are the second derivative terms $\partial^{2} E / \partial w_{i} \partial w_{j}$, is unwieldy. We have derived the $\mathcal{R}\{\cdot\}$ technique, which directly computes $\mathbf{H v}$, the product of the Hessian with a vector. The technique is

- exact: no approximations are made.

- numerically accurate: there is no drastic loss of precision.

- efficient: it takes about the same amount of computation as a gradient calculation.

- flexible: it applies to all existing gradient calculation procedures.

- robust: if the gradient calculation gives an unbiased estimate of $\nabla_{\mathbf{w}}$, then our procedure gives an analogous unbiased estimate of $\mathbf{H v}$.

Procedures that result from the applications of the $\mathcal{R}\{\cdot\}$ technique are about as local, parallel, and efficient as the original untransformed gradient calculation. The technique applies naturally to backpropagation networks, recurrent networks, relaxation networks, Boltzmann Machines, and perturbative methods. Hopefully, this new class of algorithms for efficiently multiplying vectors by the Hessian will facilitate the construction of algorithms that are efficient in both space and time.

\section{Acknowledgments}

I thank Yann le Cun and Patrice Simard for their encouragement and generosity. Without their work and enthusiasm I would not have derived the $\mathcal{R}\{\cdot\}$ technique or recognized its importance. Thanks also go to Nandakishore Kambhatla, John Moody, Thomas Petsche, Steve Rehfuss, Akaysha Tang, David Touretzky, Geoffery Towell, Andreas Weigend and an anonymous reviewer for helpful comments and careful readings. This work was partially supported by grants NSF ECS-9114333 and ONR N00014-92-J-4062 to John Moody, and by Siemens Corporate Research.

\section{References}

Ackley, D. H., Hinton, G. E., and Sejnowski, T. J. (1985). A learning algorithm for Boltzmann Machines. Cognitive Science, 9:147-169.

Almeida, L. B. (1987). A learning rule for asynchronous perceptrons with feedback in a combinatorial environment. In (Caudill and Butler, 1987), pages 609-618. 
Alspector, J., Meir, R., Yuhas, B., and Jayakumar, A. (1993). A parallel gradient descent method for learning in analog VLSI neural networks. In (Hanson et al., 1993), pages 836-844.

Becker, S. and le Cun, Y. (1989). Improving the convergence of back-propagation learning with second order methods. In Touretzky, D. S., Hinton, G. E., and Sejnowski, T. J., editors, Proceedings of the 1988 Connectionist Models Summer School. Morgan Kaufmann. Also published as Technical Report CRG-TR-88-5, Department of Computer Science, University of Toronto.

Bishop, C. (1992). Exact calculation of the Hessian matrix for the multilayer perceptron. Neural Computation, 4(4):494501.

Buntine, W. and Weigend, A. (1991). Calculating second derivatives on feedforward networks. IEEE Transactions on Neural Networks. In submission.

Caudill, M. and Butler, C., editors (1987). IEEE First International Conference on Neural Networks, San Diego, CA.

Cauwenberghs, G. (1993). A fast stochastic error-descent algorithm for supervised learning and optimization. In (Hanson et al., 1993), pages 244-251.

Flower, B. and Jabri, M. (1993). Summed weight neuron perturbation: An $O(n)$ improvement over weight perturbation. In (Hanson et al., 1993), pages 212-219.

Hanson, S. J., Cowan, J. D., and Giles, C. L., editors (1993). Advances in Neural Information Processing Systems 5. Morgan Kaufmann.

Hassibi, B. and Stork, D. G. (1993). Second order derivatives for network pruning: Optimal brain surgeon. In (Hanson et al., 1993), pages 164-171.

Hinton, G. E. (1987). Connectionist learning procedures. Technical Report CMU-CS-87-115, Carnegie Mellon University, Pittsburgh, PA 15213.

Jabri, M. and Flower, B. (1991). Weight perturbation: An optimal architecture and learning technique for analog VLSI feedforward and recurrent multilayer networks. Neural Computation, 3(4):546-565.

Kirk, D. B., Kerns, D., Fleischer, K., and Barr, A. H. (1993). Analog VLSI implementation of gradient descent. In (Hanson et al., 1993), pages 789-796.

le Cun, Y., Denker, J. S., and Solla, S. A. (1990). Optimal brain damage. In Touretzky, D. S., editor, Advances in Neural Information Processing Systems 2, pages 598-605. Morgan Kaufmann.

le Cun, Y., Kanter, I., and Solla, S. A. (1991). Second order properties of error surfaces: Learning time and generalization. In Lippmann, R. P., Moody, J. E., and Touretzky, D. S., editors, Advances in Neural Information Processing Systems 3, pages 918-924. Morgan Kaufmann.

le Cun, Y., Simard, P. Y., and Pearlmutter, B. A. (1993). Automatic learning rate maximization by on-line estimation of the Hessian's eigenvectors. In (Hanson et al., 1993), pages 156-163.

MacKay, D. J. C. (1991). A practical Bayesian framework for back-prop networks. Neural Computation, 4(3):448-472.

Møller, M. (1993a). Exact calculation of the product of the Hessian matrix of feed-forward network error functions and a vector in $O(n)$ time. Daimi PB-432, Computer Science Department, Aarhus University, Denmark.

Møller, M. (1993b). A scaled conjugate gradient algorithm for fast supervised learning. Neural Networks, 6(4):525-533.

Møller, M. (1993c). Supervised learning on large redundant training sets. International Journal of Neural Systems, 4(1).

Moody, J. E. (1992). The effective number of parameters: an analysis of generalization and regularization in nonlinear learning systems. In (Moody et al., 1992), pages 847-854.

Moody, J. E., Hanson, S. J., and Lippmann, R. P., editors (1992). Advances in Neural Information Processing Systems 4. Morgan Kaufmann.

Movellan, J. R. and McClelland, J. L. (1991). Learning continuous probability distributions with the contrastive Hebbian algorithm. Technical Report PDP.CNS.91.2, Carnegie Mellon University Dept. of Psychology, Pittsburgh, PA.

Pearlmutter, B. A. (1992). Gradient descent: Second-order momentum and saturating error. In (Moody et al., 1992), pages $887-894$.

Pineda, F. (1987). Generalization of back-propagation to recurrent neural networks. Physical Review Letters, 19(59):2229_ 2232.

Press, W. H., Flannery, B. P., Teukolsky, S. A., and Verrerling, W. T. (1988). Numerical Recipes in C. Cambridge University Press. 
Skilling, J. (1989). The eigenvalues of mega-dimensional matrices. In Skilling, J., editor, Maximum Entropy and Bayesian Methods, pages 455-466. Kluwer Academic Publishers.

Watrous, R. (1987). Learning algorithms for connectionist networks: Applied gradient methods of nonlinear optimization. In (Caudill and Butler, 1987), pages 619-627.

Werbos, P. J. (1974). Beyond Regression: New Tools for Prediction and Analysis in the Behavioral Sciences. PhD thesis, Harvard University.

Werbos, P. J. (1988). Backpropagation: Past and future. In IEEE International Conference on Neural Networks, volume I, pages 343-353, San Diego, CA.

Werbos, P. J. (1992). Neural networks, system identification, and control in the chemical process industries. In White, D. A. and Sofge, D. A., editors, Handbook of Intelligent Control-Neural, Fuzzy, and Adaptive approaches, chapter 10, pages 283-356. Van Norstrand Reinhold. see section 10.7.

Widrow, B., McCool, J. M., Larimore, M. G., and Johnson, Jr., C. R. (1979). Stationary and nonstationary learning characteristics of the LMS adaptive filter. Proceedings of the IEEE, 64:1151-1162. 